\title{
Acute renal artery stenting recovered renal function after spontaneous rupture of renal artery aneurysm - case report
}

\author{
Michał Sojka ${ }^{A, B, C, D, E, F, G}$, Maciej Szmygin ${ }^{A, B, C, D, E, F, G}$, Krzysztof Pyra ${ }^{A, B, C, D, E, E, G}$, Maryla Kuczyńska ${ }^{A, B, C, D, E, E, F, G}$, \\ Tomasz Jargiełło ${ }^{A, B}, C, D, E, F, G$
}

Department of Interventional Radiology and Neuroradiology, Medical University of Lublin, Lublin, Poland

\section{Abstract}

Purpose: Renal artery aneurysms (RAAs) are a rare vascular pathology with an estimated prevalence of $0.1 \%$ to $2.5 \%$. Rupture of RAA is an extremely unusual cause of acute flank pain and haemodynamic instability with acute kidney failure and high mortality rate (20\%).

Case report: A 37-year-old male with no relevant history presented to the Emergency Room with acute right flank pain. Initial examination revealed BP $90 / 60 \mathrm{~mm} \mathrm{Hg}$ and tachycardia. Initial blood testing was unremarkable, with a haemoglobin level of $9.4 \mathrm{~g} / \mathrm{dl}$. Urinalysis revealed moderate blood. Ultrasound (US) examination depicted aneurysm of the right renal artery $6 \times 6 \mathrm{~cm}$, with signs of blood extravasation visible in colour and power Doppler. The patient was referred for urgent computed tomography angiography, which revealed active bleeding from the ruptured aneurysm with haematoma spreading into the right retroperitoneum. He was subjected to emergency endovascular treatment. The patient was treated by successful implantation of a Viabahn stent (GORE, Daleware, USA). Selective nephrography revealed lack of flow through one of the segmental arteries resulting from vasospasm due to the placement of the guiding wire necessary for safe stent implantation.

Conclusions: The authors present a rare case of spontaneous RAA rupture in a young male successfully treated with endovascular methods. Stent implantation required selective catheterisation of segmental arteries of the kidney, which resulted in the loss of one of them. However, control Doppler US disclosed no ischaemia and successful exclusion of the aneurysm.

Key words: rupture, endovascular aneurysm repair, stent.

\section{Introduction}

Renal artery aneurysms (RAAs) are a rare vascular pathology with an estimated prevalence of $0.1 \%$ to $2.5 \%$ [1]. Rupture of RAA is an extremely unusual cause of acute flank pain and haemodynamic instability with acute kidney failure and high mortality rate (20\%) [2].

The authors present a case of a 37-year-old male with ruptured right renal artery aneurysm successfully treated by endovascular means.

\section{Case report}

A 37-year-old male with no relevant history presented to the Emergency Room with acute right flank pain. Initial examination revealed BP 90/60 mm $\mathrm{Hg}$ and tachycardia. Initial blood testing was unremarkable, with haemoglobin level of $9.4 \mathrm{~g} / \mathrm{dl}$. Urinalysis revealed moderate blood. Ultrasound (US) examination depicted an aneurysm of the right renal artery of dimensions $6 \times 6 \mathrm{~cm}$, with signs of blood extravasation visible in colour and power Doppler.

\section{Correspondence address:}

Maciej Szmygin, Department of Interventional Radiology and Neuroradiology, Medical University of Lublin, 8 Jaczewskiego St., 20-954 Lublin, Poland, e-mail: mszmygin@gmail.com

Authors' contribution:

A Study design · B Data collection · C Statistical analysis · D Data interpretation - E Manuscript preparation · F Literature search · G Funds collection 

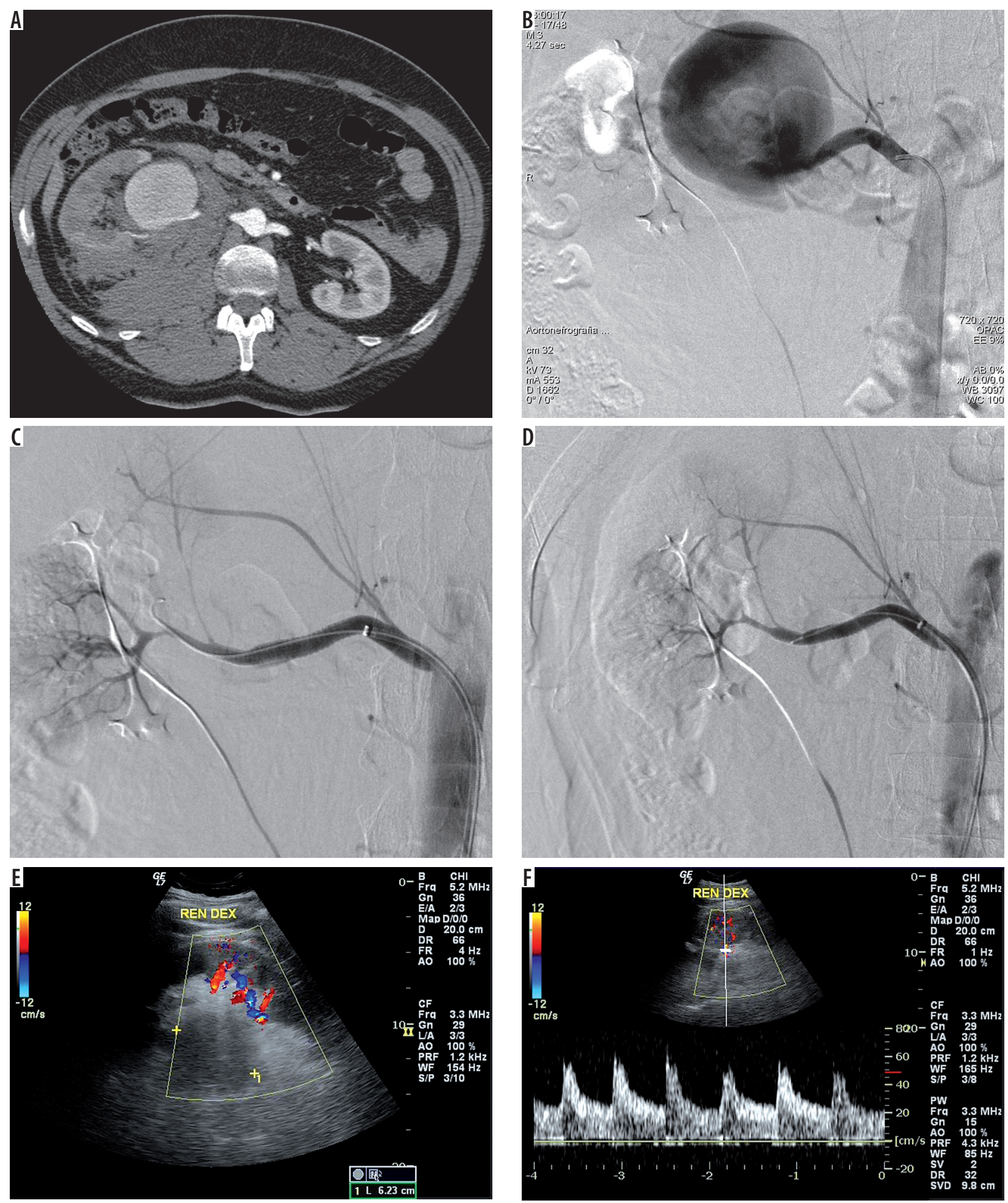

Figure 1. A) An axial computed tomography showing an aneurysm of the right renal artery. B-D) Initial angiography disclosing filling of the aneurysm of the right renal artery. C) Introduction of the stent covering the neck of the aneurysm. Tip of the guiding wire introduced into the segmental artery. D) Final nephrography revealing no filling of the aneurysmal sac. Lack of flow through one of the segmental arteries was recognised. E, F) Control Doppler ultrasound examination shows undisrupted, uniform filling of all major arterial branches with no flow to the aneurysm

The patient was referred for urgent computed tomography $(\mathrm{CT})$ angiography, which revealed active bleeding from the ruptured aneurysm with haematoma spreading into the right retroperitoneum (Figure 1A).

He was subjected to emergency endovascular treatment.
The patient was treated by successful implantation of a Viabahn stent (GORE, Daleware, USA). Selective nephrography revealed lack of flow through one of the segmental arteries resulting from vasospasm due to the placement of a guiding wire that was necessary for safe stent implantation (Figures 1B-D). 
Control Doppler US performed 24 hours after the procedure revealed undisrupted, uniform filling of all major arterial branches and no filling of the aneurysmal sac (Figures 1E, F).

After five days the patient was discharged home in good clinical condition.

\section{Discussion}

RAAs are rare entity, with an incidence of up to $2.5 \%$ in the general population $[2,3]$. The aetiology of true RAA is associated with degeneration of connective tissue (fibromuscular dysplasia, Marfan syndrome), vasculitis, and atherosclerosis [4,5]. Pseudoaneurysms of renal arteries occur in patients after trauma, infection, or inflammation.

True aneurysms are most commonly located at the renal artery trunk or at the bifurcation, and pseudoaneurysms occur mostly in the distal parts of the renal artery [6].

The majority of patients with RAAs will present with hypertension $(73 \%)$. Other typical manifestations include haematuria (30\%) and abdominal or lumbar pain (8-25\%) [7]. The treatment of renal artery aneurysms can be both surgical and percutaneous and depends on their location and morphology as well as comorbidities and the acute clinical condition of the patient. One of the biggest studies, following over 2700 patients with identified aneurysms, who underwent RAA repair, concluded that endovascular techniques are associated with shorter hospitalisation, fewer complications requiring rehabilitation, and better clinical outcome compared to conventional open surgery [8].

Spontaneous rupture or RAA is an extremely unusual cause; therefore, the number of studies available to date is very limited. The prognosis is poor, and it results most commonly in loss of the affected kidney [9]. Li et al. [10] published a unique study of six patients with ruptured RAA treated with endovascular methods. The authors emphasise that stenting of the renal artery in an emergency setting is difficult and might require the sacrifice of some branches of the renal artery.

\section{Conclusions}

The authors present a rare case of spontaneous RAA rupture in a young male, which was successfully treated with endovascular methods. Stent implantation required selective catheterisation of segmental arteries of the kidney, which resulted in the loss of one of them. However, control Doppler US disclosed no ischaemia and successful exclusion of the aneurysm. In conclusion, endovascular methods proved to be safe and effective in treating spontaneously ruptured renal artery aneurysm and should therefore be considered in such cases.

\section{Conflict of interest}

The authors declare no conflict of interest.

\section{References}

1. Wason SE, Schwaab T. Spontaneous rupture of a renal artery aneurysm presenting as gross hematuria. Rev Urol 2010; 12: e193-e196.

2. Tulsyan N, Kashyap VS, Greenberg RK, et al. The endovascular management of visceral artery aneurysms and pseudoaneurysms. J Vasc Surg 2007; 45: 276-283.

3. Zhang LJ, Yang GF, Qi J, Shen W. Renal artery aneurysm: diagnosis and surveillance with multidetector-row computed tomography. Acta Radiol 2016; 48: 274-279.

4. Eskandari MK, Resnick SA. Aneurysms of the renal artery. Semin Vasc Surg 2005; 18: 202-208.

5. Arlet JB, Marinho A, Cluzel P, Wechsler B, Piette JC. Arterial aneurysms in Wegener's granulomatosis: case report and literature review. Semin Arthritis Rheum 2008; 37: 265-268.
6. Eldem G, Erdoğan E, Peynircioğlu B, Arat A, Balkancı F. Endovascular treatment of true renal artery aneurysms: a single center experience. Diagn Interv Radiol 2019; 25: 62-70.

7. Debus ES, Grundmann RT. Evidence-Based Therapy in Vascular Surgery. Springer, Cham 2017; pp. 113-123.

8. Buck DB, Curran T, McCallum JC, et al. Management and outcomes of isolated renal artery aneurysms in the endovascular era. J Vasc Surg 2016; 63: 77-81.

9. Hageman JH, Smith R, Szilagyi E, Elliott J. Aneurysms of the renal artery: problems of prognosis and surgical management. Surgery 1978; 84: 563.

10. Li G, Sun Y, Song H, Wang Y. Embolization of ruptured renal artery aneurysms. Clin Exp Nephrol 2015; 19: 901-908. 